\title{
Special issue: Selected and revised papers from the 18th International Conference of the Italian Association for Artificial Intelligence
}

\author{
Mario Alviano ${ }^{\mathrm{a}, *}$, Gianluigi Greco ${ }^{\mathrm{a}}$ and Francesco Scarcello ${ }^{\mathrm{b}}$ \\ ${ }^{a}$ Department of Mathematics and Computer Science, University of Calabria, Rende (CS), Italy \\ ${ }^{\mathrm{b}}$ Department of Computer Science, Modelling, Electronics and Systems Engineering University of Calabria, \\ Rende (CS), Italy
}

The Italian Association for Artificial Intelligence (AIxIA) was established in 1988 and has the role of increasing the knowledge of Artificial Intelligence (AI) at several levels, by fostering its teaching in schools and universities, and by promoting both theoretical and applied research in the field through seminars, targeted initiatives and sponsorship of events. AIxIA is a member of the European Association for Artificial Intelligence (EurAI), and has witnessed and supported the entire evolution of AI: from the initial works and expectations in the 80s, passing through a maturation period in the 90s, up to the recent success of several AI technologies pervasively used in everyday applications, AIXIA has been a center of aggregation and fertilization of ideas for the Italian and international research in the sector.

Since 1989, AIxIA organizes its International Conference, which is attended by a lively community interested in AI research, tasks, and applications. Experts of AI meet and exchange ideas and results, discuss their positions and envisage future developments of the discipline. Originally a bi-annual event, since 2014 the International Conference of the AIxIA is held annually, a change resulting from the necessity to match the rapid advances in AI. Targeting a var-

${ }^{*}$ Corresponding author: Mario Alviano, Department of Mathematics and Computer Science, University of Calabria, Via Bucci 30/B, 87036 Rende (CS), Italy. E-mail: mario.alviano@unical.it. ied audience of people interested in AI, the format of the International Conference includes, in addition to the main track and invited talks, several poster sessions, a number of workshops (some focused on research topics, others on applications), tutorials, and a Doctoral Consortium. Additionally, a novelty for this edition of the conference was the presentation of discussion papers about recently published research. The program is often complemented by an open event targeted to the general public concerning the impact of AI on society, and this edition of the conference focused on trusting people with AI technologies.

The 18th International Conference of the Italian Association for Artificial Intelligence (AIxIA 2019) was held in Rende (CS), Italy, from November 19 to November 22, 2019. The conference was attended by around 200 delegates, received 86 submissions, 67 of which selected for the technical review process. The Program Committee decided to accept 41 papers for the main track of the conference. Among these, the General and Programme Chairs identified a set of papers that received particularly good reviews and selected five of them for inclusion in this special issue after a further review process:

- Prioritized Multi-Criteria Federated Learning, by Vito Walter Anelli, Yashar Deldjoo, Tommaso Di Noia and Antonio Ferrara, proposing new client-specific criteria for Federated 
Learning, a recent Machine Learning approach comprising a federation of users asked to train a global model based on their private data, and a central coordinating server aggregating locally computed updates by clients.

- Adversarial Training for Few-Shot Text Classification, by Danilo Croce, Giuseppe Castellucci and Roberto Basili, proposing the use of SemiSupervised Generative Adversarial Networks to boost the performances of simple architectures for Natural Language Processing.

- Proof-search and countermodel generation for non-normal modal logics: the theorem prover PRONOM, by Tiziano Dalmonte, Sara Negri, Nicola Olivetti and Gian Luca Pozzato, presenting a theorem prover and countermodel generator for non-normal modal logics based on labelled sequent calculi.

- AI for interactive performance: challenges and techniques, by Rossana Damiano, Vincenzo Lombardo, Antonio Pizzo and Giulia Monticone, analyzing the contribution of AI techniques in the design and realization of a dramatic performance, an interactive system participated by human performers and audiences through some type of enactment.

- Text classification by untrained sentence embeddings, by Daniele Di Sarli, Claudio Gallicchio and Alessio Micheli, addressing the question of whether it is possible to generate sentence embeddings via completely untrained recurrent dynamics, on top of which to apply a simple learning algorithm for text classification.

We would like to thank all members of the AIxIA 2019 Programme Committee for their effort and invaluable contribution to the review process, that was fundamental to the high scientific level of the event, and the additional reviewers recruited for the special issue reviews. We also would like to thank the AIxIA Executive Board, and all the researchers of the Artificial Intelligence community who supported this event by submitting their work and actively participating in it. Last but not least, we are grateful to Fabrizio Riguzzi, Editor in Chief of Intelligenza Artificiale, for hosting this special issue.

\section{AIxIA 2019 Programme Committee Members}

- Fabrizio Angiulli, University of Calabria, Italy

- Davide Bacciu, University of Pisa, Italy
- Farshad Badie, Aalborg University, Denmark

- Matteo Baldoni, University of Turin, Italy

- Stefania Bandini, CSAI - Complex Systems and Artificial Intelligence Research Center, Italy

- Roberto Basili, University of Roma Tor Vergata, Italy

- Nicola Basilico, University of Milan, Italy

- Federico Bergenti, University of Parma, Italy

- Tarek Richard Besold, Alpha Health AI Lab, Telefonica Innovation Alpha, Germany

- Stefano Bistarelli, University of Perugia, Italy

- Andrea Burattin, Technical University of Denmark, Denmark

- Elena Cabrio, Université Côte d'Azur, CNRS, CNRS, Inria, I3S, France

- Stefano Cagnoni, University of Parma, Italy

- Marco Calautti, University of Calabria, Italy

- Diego Calvanese, Free University of BozenBolzano, Italy

- Luigia Carlucci Aiello, Sapienza University of Rome, Italy

- Amedeo Cesta, CNR - National Research Council of Italy, Italy

- Federico Chesani, University of Bologna, Italy

- Francesco Corcoglioniti, Fondazione Bruno Kessler, Italy

- Gabriella Cortellessa, CNR-ISTC, Italy

- Stefania Costantini, University of 1'Aquila, Italy

- Dario Della Monica, University of Udine, Italy

- Claudio Di Ciccio, Vienna University of Economics and Business, Austria

- Tommaso Di Noia, Polytechnic University of Bari, Italy

- Carmine Dodaro, University of Calabria, Italy

- Michele Donini, Amazon, USA

- Agostino Dovier, University of Udine, Italy

- Aldo Franco Dragoni, Polytechnic University of Marche, Italy

- Stefano Ferilli, University of Bari, Italy

- Johannes Fichte, TU Dresden, Germany

- Alberto Finzi, University of Naples Federico II, Italy

- Giancarlo Fortino, University of Calabria, Italy

- Salvatore Gaglio, University of Palermo, Italy

- Marco Gavanelli, University of Ferrara, Italy

- Chiara Ghidini, Fondazione Bruno Kessler, Italy

- Massimiliano Giacomin, University of Brescia, Italy

- Laura Giordano, Università del Piemonte Orientale, Italy 
- Giuseppe Jurman, Fondazione Bruno Kessler, Italy

- Evelina Lamma, University of Ferrara, Italy

- Domenico Lembo, Sapienza University of Rome, Italy

- Antonio Lieto, University of Turin, Italy

- Marco Lippi, University of Modena and Reggio Emilia, Italy

- Francesca Alessandra Lisi, University of Bari, Italy

- Fabrizio Maria Maggi, Institute of Computer Science - University of Tartu, Estonia

- Bernardo Magnini, FBK-irst, Italy

- Marco Manna, University of Calabria, Italy

- Marco Maratea, University of Genoa, Italy

- Simone Marinai, University of Florence, Italy

- Andrea Marrella, Sapienza University of Rome, Italy

- Viviana Mascardi, University of Genoa, Italy

- Fulvio Mastrogiovanni, University of Genoa, Italy

- Alessandro Mazzei, University of Turin, Italy

- Stefano Melacci, University of Siena, Italy

- Paola Mello, University of Bologna, Italy

- Alessio Micheli, University of Pisa, Italy

- Alfredo Milani, University of Perugia, Italy

- Stefania Montani, University of Piemonte Orientale, Italy

- Angelo Oddi, ISTC-CNR, Italy

- Matteo Palmonari, University of MilanoBicocca, Italy

- Andrea Passerini, University of Trento, Italy

- Viviana Patti, University of Turin, Italy

- Ruggero G. Pensa, University of Turin, Italy

- Roberto Pirrone, University of Palermo, Italy

- Piero Poccianti, Consorzio Operativo Gruppo MPS, Italy

- Daniele Porello, Free University of BolzanoBozen, Italy

- Gian Luca Pozzato, University of Turin, Italy

- Fabrizio Riguzzi, University of Ferrara, Italy

- Andrea Roli, University of Bologna, Italy

- Riccardo Rosati, Sapienza University of Rome, Italy

- Silvia Rossi, University of Naples Federico II, Italy

- Salvatore Ruggieri, University of Pisa, Italy

- Emanuel Sallinger, University of Oxford, UK

- Fabio Sartori, University of Milan-Bicocca, Italy

- Marco Schaerf, Sapienza University Rome, Italy
- Giovanni Semeraro, University of Bari, Italy

- Luciano Serafini, Fondazione Bruno Kessler, Italy

- Domenico Talia, University of Calabria, Italy

- Stefano Teso, Katholieke Universiteit Leuven, Belgium

- Mauro Vallati, University of Huddersfield, UK

- Eloisa Vargiu, Eurecat Technology Center eHealth Unit, Spain

- Marco Villani, University of Modena and Reggio Emilia, Italy

- Giuseppe Vizzari, University of MilanoBicocca, Italy

- Antonius Weinzierl, Vienna University of Technology, Austria

\section{AIxIA 2019 Additional Reviewers}

- Sebastia Agramunt

- Agnese Augello

- Elena Bellodi

- Alessandro Bertagnon

- Federico Bianchi

- Giulio Biondi

- Mattia Cerrato

- Francesco Crecchi

- Alessandro Dal Palù

- Riccardo De Benedictis

- Alessandra De Paola

- Giovanni Ercolano

- Francesca Fracasso

- Massimo La Rosa

- Antonio Lieto

- Salim Malek

- Fedelucio Narducci

- Tobias Philipp

- Mirko Polato

- Marco Polignano

- Williams Rizzi

- Luca Romeo

- Francesco Santini

- André Schidler

- Carlo Taticchi

- Francesco Tiezzi

- Sahar Vahdati
Rende, 30 September 2020 Mario Alviano, Gianluigi Greco and Francesco Scarcello 